\title{
Venous thromboembolism in non-COVID-19 population during the pandemic: a nationwide multicenter retrospective survey
}

\author{
Cheng Qian ${ }^{1} \cdot \mathrm{XiaO}_{\mathrm{Lyu}}{ }^{2} \cdot \mathrm{Hai}-$ Dong $\mathrm{Zhu}^{2} \cdot \mathrm{Yi} \mathrm{Zhang}^{2} \cdot$ Rui-Jie $\mathrm{Du}^{2} \cdot \mathrm{Rui} \mathrm{Li}^{2} \cdot$ Gao-Jun Teng ${ }^{2}$. Jian-Ping Gu${ }^{1}$
}

Accepted: 30 March 2021 / Published online: 14 May 2021

(C) The Author(s), under exclusive licence to Springer Science+Business Media, LLC, part of Springer Nature 2021

\begin{abstract}
Impact of pandemic on the incidence of venous thromboembolism (VTE) in non-COVID-19 patients is undetermined. Thus, a nationwide multicenter retrospective survey was conducted to evaluate the disease burden in non-COVID-19 population. This multi-center survey involved 94 hospitals from 24 provinces in the mainland of China, and collected data on nonCOVID-19 patients admitted to the radiology departments due to VTE between January 24 and April 16, 2020. Baseline characteristics, VTE risk factors, clinical manifestations and the treatments were compared with those in the same period of 2019. 3,358 patients with VTE from 74 hospitals were included in this study (1,458 in 2020, 1,900 in 2019). Most aged $\geq 50$ years $(80.6 \%$ in the pandemic, $81.2 \%$ in 2019). The number of patients aged 30-39 years increased from 3.9\% in 2019 period to $5.8 \%$ in the pandemic $(p=0.009)$. Among the VTE risk factors, the rate of decreased activity increased significantly in the pandemic, and was much higher than that in 2019 (30.7\% vs $22.6 \%, p<0.0001)$. Under the risk of decreased activity, patients with comorbidities chronic diseases, especially diabetes, showed significantly a higher incidence of VTE $(30.4 \%$ vs $22.0 \%, p<0.0001)$. In the pandemic period, fewer patients were treated with anticoagulation alone (33.5\% vs $36.7 \%$, $\mathrm{p}=0.05$ ), and more underwent inferior vena cava filter (IVCF) implantation, compared with those in 2019 (66.5\% vs $63.2 \%$, $\mathrm{p}=0.046$ ). The pandemic increased the VTE risk of decreased activity among the non-COVID-19 population. Patients with comorbidities, especially diabetes, have a significant higher risk of VTE during the pandemic.
\end{abstract}

Keywords Coronavirus disease 2019 $\cdot$ Venous thromboembolism $\cdot$ Non-COVID-19 population $\cdot$ Risk factors $\cdot$ Decreased activity

\section{Highlights}

- The pandemic affected the risk factors of venous thromboembolism (VTE).

Cheng Qian, Xiao Lyu and Hai-Dong Zhu contributed equally to this work.

Gao-Jun Teng

gjteng@vip.sina.com

$\triangle$ Jian-Ping Gu

cjr.gujianping@vip.163.com

1 Department of Interventional Radiology, Nanjing First Hospital, 68 Changle Rd., Nanjing, Jiangsu Province, China

2 Center of Intervention and Vascular Surgery, Department of Radiology, Zhongda Hospital, Southeast University, 87 Dingjiaqiao Rd., Nanjing 210009, Jiangsu Province, China
- Decreased activity is the leading VTE risk factor among the non-COVID-19 population.

- Patients with comorbidities, especially diabetes, showed a significant higher risk of VTE.

\section{Introduction}

The coronavirus disease 2019 (COVID-19) is highly contagious and has caused a rapid global pandemic, leading to ten millions infected [1]. Hemostatic abnormalities were reported in patients with COVID-19, such as elevated D-dimer levels ( $>1 \mathrm{~g} / \mathrm{L})$, prolonged prothrombin time, and increased fibrin degradation (FDP) level [2]. In a multicenter retrospective cohort study, COVID-19 cases with elevated D-dimer levels were reported a highly in-hospital death [3]. In another study, COVID-19 non-survivors showed significantly higher D-dimer and FDP levels [4]. And concomitant venous thromboembolism (VTE), comprising deep vein 
thrombosis (DVT) and pulmonary embolism (PE), has been frequently reported in these patients. A study including 81 COVID-19 cases in China, reported 25\% developed VTE in intensive care unit [5]. Meanwhile, another study from Netherlands reported that, among 184 patients with severe COVID-19, 31\% developed incident VTE (95\% CI 20-41) [6].

All the above-mentioned studies focused on patients with COVID-19, and demonstrated that the COVID-19 patients were more likely to have an incident of VTE. However, non-COVID-19 population was far larger than COVID-19 patients. Few attentions have been paid to non- COVID19 people during the pandemic. The social distancing and self-isolation, and the change of lifestyle, may influence the prevalence of VTE in non-COVID-19 patients. Hence, this nationwide multi-center investigation was conducted on nonCOVID-19 patients admitted to the radiology departments in China and designed to evaluate the prevalence, the characterize clinical presentation and the potential risk factors for VTE in non-COVID-19 patients.

\section{Patients and methods}

This multi-center survey involving 94 radiology departments from 24 provinces in the mainland of China, was conducted between January 24 and April 16, 2020 (from the locking down of Wuhan to work resumption in most cities across China). This survey was approved by each institutional review board. Non-COVID-19 patients admitted to the radiology departments due to VTE were enrolled. Baseline characteristics (age, sex, and comorbidities disease), VTE risk factors, and clinical manifestations were collected by a questionnaire (Supplemental table). Except for age, all other questions have been presented in the form of multiple-choice questions. Possible VTE risk factors include decreased activity (greater time spent sitting or lying down, and physical activity time less than half of the usual $\geq 3$ days, with no other disease reasons), recent surgery performed in the prior 2 months (including abdominal, cardiac, neurological surgery, etc.), traumatic fractures without surgery in the prior one month, immobilization for at least one week for any nonsurgical reason in the prior one month and others (including hormonal therapy, fat and chronic lung disease) [7, 8]. The categories of VTE including DVT and PE were analyzed. The treatments were also evaluated, including anticoagulation alone, inferior vena cava filter (IVCF) implantation, and additional interventional radiology therapy (including catheter-directed thrombolysis, percutaneous mechanical thrombectomy, balloon dilatation, or stent implantation). Data were compared with those of the in-hospital VTE patients during the same period in 2019 (from January 24 to April 16). Finally, three senior doctors gathered and analyzed all the data.

\section{Statistical analysis}

Individual data were summarized as frequencies or percentages for categorical variables and median (interquartile range) for continuous variables. The Chi-square test was used to compare categorical variables. Student's t-test was used to compare continuous variables. The differences were considered statistically significant at $p \leq 0.05$ (two-tailed). All statistical analyses were performed using SPSS software (version 22).

\section{Results}

\section{Demographics}

Among the 94 hospitals, four had no data in the pandemic period, sixteen did not provide the data of the same period in 2019, and three cases were with COVID-19. Finally, a total of 3,358 patients from 74 hospitals across the mainland China were included in this study (Fig. 1). During the pandemic period (between January 24 and April 16, 2020), 1,458 non-COVID-19 patients were diagnosed with VTE, with 745 males $(51.1 \%)$, the median age was 64 years. During the same period in 2019, 1,900 patients were diagnosed with VTE, with 915 males (48.2\%), the median age was 64 years. No statistical differences were observed between the two groups $(p>0.05)$ (Table 1).

In both groups, the rate of VTE increased with aging and reached a peak at the age of 60-69 years. The incidence of VTE was mostly in the patients aged $\geq 50$ years, $80.6 \%$ in the pandemic period, and $81.2 \%$ in the same period of 2019 . In the pandemic period, more patients aged $30-39$ years had an incident VTE than those in 2019 period (5.8\% vs 3.9\%, $p=0.009$ ) (Fig. 2).

\section{Chronic comorbidities}

During the pandemic period, a total of 724 patients (49.7\%) were with chronic comorbidities, and 334 (23.6\%) with more than two kinds of comorbidities. The most common diseases were hypertension (34.0\%), diabetes (18.2\%), coronary artery disease $(12.2 \%)$ and stroke $(11.5 \%)$ in the pandemic period. Others were $11.8 \%$, including chronic obstructive pulmonary disease, chronic kidney disease, etc. During the same period in 2019 , hypertension (34.7\%), diabetes $(15.4 \%)$, stroke (10.5\%) and coronary artery disease (10.3\%) remained the most common comorbidities. Patients with diabetes showed significantly a higher incidence of VTE during 
Fig. 1 Flow diagram

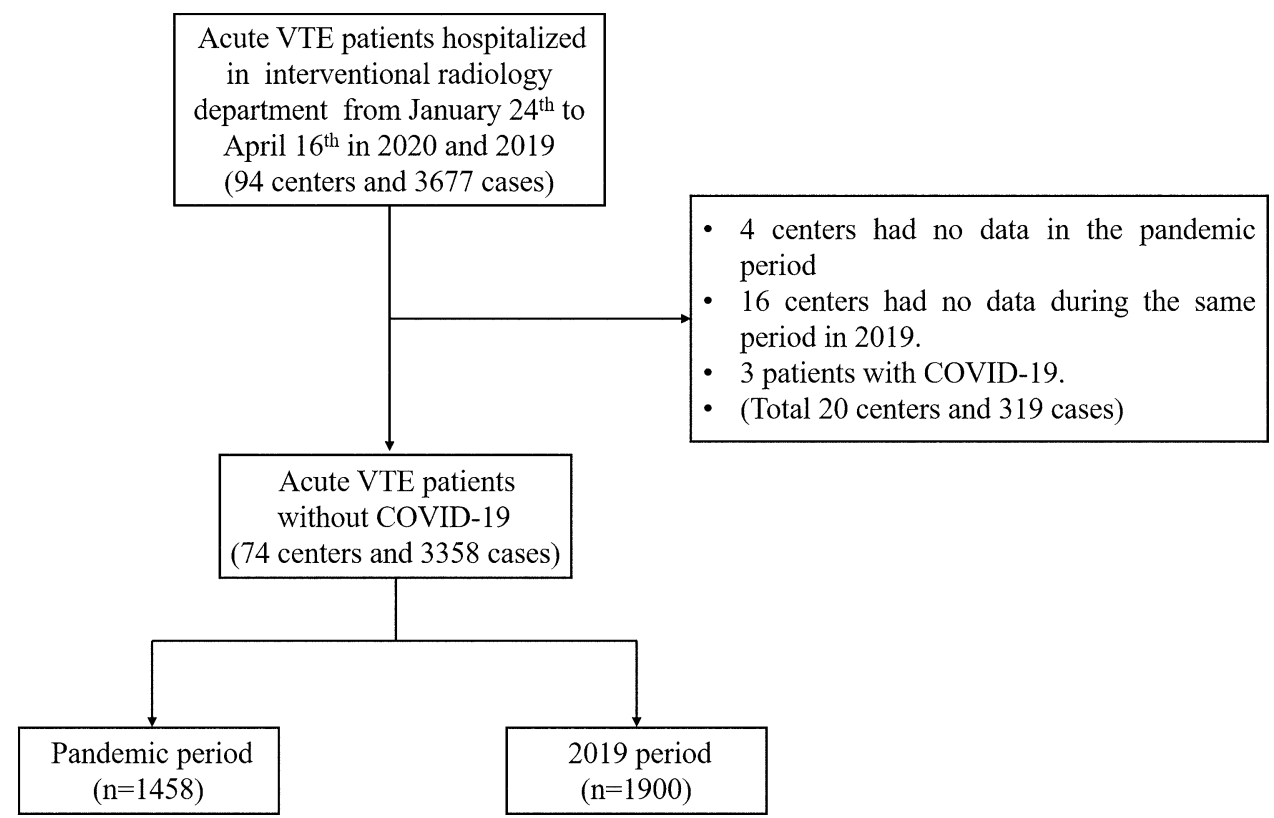

the pandemic period than those in the same period of 2019 (18.2\% vs $15.4 \%, p<0.001)$ (Table 1$)$.

\section{Risk factors}

Among the potential risk factors of VTE, decreased activity was the first factor in the pandemic period, and the rate was significantly higher than that in the same period of 2019 (30.7\% vs $22.6 \%, p<0.0001)$. Patients with more than two risk factors showed significantly a higher incidence of VTE than those in 2019 (5.6\% vs $4.1 \%, p=0.041)$. Under the risk of decreased activity, patients with comorbidities chronic diseases showed more likely to have an incident VTE than those without comorbidities chronic diseases $(30.4 \%$ vs $22.0 \%, p<0.0001)$. However, the incidence rate of VTE due to trauma decreased from $13.1 \%$ in 2019 period to $11.5 \%$ in pandemic period, and the incidence rate of VTE induced by surgery was also lower during the pandemic period $(8.4 \%$ vs $11.3 \%, p=0.007)$. The remaining risk factors such as active cancer, immobilization, previous VTE, and so on showed no significant differences between the two groups $(p>0.05)$ (Table 1).

\section{Clinical manifestations}

The clinical manifestations such as syncope, stuffiness, swelling, lower limb pain, or tenderness showed no significant differences between the two groups. Most patients admitted to the hospitals with limb symptoms alone, $89 \%$ in the pandemic period and $88 \%$ in the same period of 2019 . In the pandemic period, 1,142 (78.3\%) patients with DVT alone, 26 (1.8\%) with PE alone, and 290 (19.9\%) with PE and DVT, versus 1,494 (78.6\%), 40 (2.1\%) and $366(19.3 \%)$ in the same period of 2019, respectively. No statistical differences were observed between the two groups. The total rate of incidence PE was $21.7 \%$ in the pandemic period, versus $21.4 \%$ in the same period of 2019 . And the rate of symptomatic PE was also similar between the groups $(43.4 \%$ vs $48.0 \%, p>0.05)$. The number of patients treated with anticoagulation alone during the pandemic period was fewer than that in $2019(33.5 \%$ vs $36.7 \%, p=0.05)$. And more patients accepted IVCF implantation in the pandemic period $(66.5 \%$ vs $63.2 \%, p=0.046)$. The rate of additional interventional therapy also increased from $41.9 \%$ to $44.1 \%$ in the pandemic period. The short-outcome on discharge showed no difference between two groups (Table 2).

\section{Discussions}

This is a nationwide survey to provide insights into the disease burden of VTE in the non-COVID-19 population. Among the VTE patients without COVID-19, 80.6\% aged $\geq 50$ years during the pandemic. Population-based studies reported that the incidence of VTE rises exponentially with age, and $>60 \%$ VTE events occur in persons aged $\geq 65$ years $[9,10]$. Those patients have higher mortality and VTErelated morbidity than younger patients, including anticoagulation-related bleeding and the post-thrombotic syndrome [11]. Therefore, special attention should be paid to elder non-COVID-19 patients during the pandemic.

As the world is entering into increasingly unprecedented territory with lockdowns and social-distancing orders, nonCOVID-19 patients are asked to self-isolation [12]. Many 
Table 1 Baseline characteristics of patients with venous thromboembolism in the pandemic period and in the same period of 2019

\begin{tabular}{|c|c|c|c|}
\hline & Pandemic $(\mathrm{n}=1,458)$ & $2019(\mathrm{n}=1900)$ & $p$ value \\
\hline Male, n (\%) & $745(51.1)$ & $915(48.2)$ & 0.091 \\
\hline Age(years), median (IQR) & $64(53-72)$ & $64(53-73)$ & 0.258 \\
\hline \multicolumn{4}{|l|}{ Chronic comorbidities } \\
\hline Hypertension, n (\%) & $495(34.0)$ & $659(34.7)$ & 0.657 \\
\hline Diabetes, n (\%) & $266(18.2)$ & $292(15.4)$ & $0.026 *$ \\
\hline Coronary artery disease, $\mathrm{n}(\%)$ & $178(12.2)$ & $195(10.3)$ & 0.075 \\
\hline Stroke, n (\%) & $168(11.5)$ & $200(10.5)$ & 0.360 \\
\hline Others & $172(11.8)$ & $206(10.8)$ & 0.386 \\
\hline Number of chronic diseases & & & 0.637 \\
\hline $0, \mathrm{n}(\%)$ & $734(50.3)$ & $976(51.4)$ & \\
\hline $1, \mathrm{n}(\%)$ & $380(26.1)$ & $502(26.4)$ & \\
\hline$\geq 2, \mathrm{n}(\%)$ & $344(23.6)$ & $422(22.2)$ & \\
\hline \multicolumn{4}{|l|}{ Risk factors } \\
\hline Decreased activity, $\mathrm{n}(\%)^{\mathrm{a}}$ & $448(30.7)$ & $430(22.6)$ & $<0.001$ \\
\hline Active cancer, n (\%) & $200(13.7)$ & $278(14.7)$ & 0.427 \\
\hline Trauma, n $(\%)^{\mathrm{b}}$ & $168(11.5)$ & $249(13.1)$ & 0.168 \\
\hline Surgery, $\mathrm{n}(\%)^{\mathrm{c}}$ & $123(8.4)$ & $214(11.3)$ & 0.007 \\
\hline Immobilization, $\mathrm{n}(\%)^{\mathrm{d}}$ & $124(8.5)$ & $144(7.6)$ & 0.326 \\
\hline Previous VTE, n (\%) & $95(6.5)$ & $103(5.4)$ & 0.182 \\
\hline Pregnancy or puerperium, n (\%) & $24(1.6)$ & $31(1.6)$ & 0.974 \\
\hline Autoimmune diseases, n (\%) & $12(0.8)$ & $17(0.9)$ & 0.824 \\
\hline Hematological diseases, $\mathrm{n}(\%)$ & $19(1.3)$ & $31(1.6)$ & 0.436 \\
\hline Travel > $6 \mathrm{~h}, \mathrm{n}(\%)$ & $7(0.5)$ & $8(0.4)$ & 0.799 \\
\hline Others or none, $\mathrm{n}(\%)^{\mathrm{e}}$ & $352(24.1)$ & $515(27.1)$ & 0.052 \\
\hline$\geq$ Two risk factors, $\mathrm{n}(\%)$ & $81(5.6)$ & $77(4.1)$ & 0.041 \\
\hline
\end{tabular}

$I Q R$ interquartile range, VTE venous thromboembolism

${ }^{a}$ Decreased activity (greater time spent sitting or lying down, and physical activity time less than half of the usual $\geq 3$ days)

${ }^{\mathrm{b}}$ Recent trauma without surgery

${ }^{\mathrm{c}}$ Recent surgery performed in the prior 2 months (including trauma, abdominal, genitourinary, orthopedic, cardiac, vascular, or neurological surgery)

${ }^{\mathrm{d}}$ Immobilization for at least 7 days for any non-surgical reason in the prior one month (associated or not with medical conditions, or non-operable fractures)

${ }^{\mathrm{e}}$ Other risk factors including hormonal therapy, fat and chronic lung disease people spent more time sitting or lying down at home, and their physical activity time was less than half of the usual [13]. The present study showed that decreased activity was the first risk factor in the pandemic period, with significantly higher rate than that in the same period of 2019 . Decreased activity reduces venous blood flow, particularly in the pockets of the venous valves, resulting in stasis and increased coagulation [14]. It also increases the possibility of prethrombotic state when the duration is more than $24 \mathrm{~h}$, it will be a risk factor if the duration exceeds 3 days [15]. In previous studies, decreased activity has been identified as the main factor of hospital-acquired VTE [16]. Data from the RIETE registry trial reported that recent decreased activity provoke the recurrence of VTE and PE in COPD patients with VTE, the OR are $0.96(0.61-1.53)$ and $1.01(0.57-1.79)$ respectively [15]. Therefore, this study recommended appropriate activity to reduce the possibility of VTE during the self-isolation. On the other hand, decreased activity reduced the risk of trauma and surgery in turn. Thus, the pandemic also affected the constituent ratio of the VTE risk factors.

Under the risk of decreased activity, patients with chronic comorbidities disease, especially diabetes, showed a higher incident VTE rate. The linking between diabetes and VTE has been proven in some studies. It has been reported that many clotting factors are elevated in diabetes mellitus [17]. And a comprehensive meta-analysis also suggested that diabetes was associated with increased risk of VTE [18]. Therefore, patients with chronic comorbidities disease should be aware of incident VTE during the pandemic.During the pandemic, home-based exercise can be beneficial for older adults [19, 20]. Behnood Bikdeli et al. recommended the 


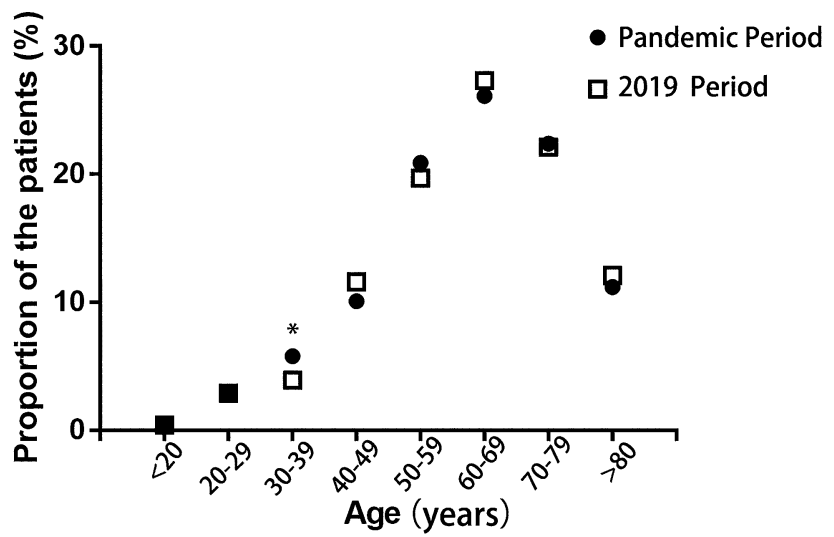

Fig. 2 Proportion of the patients with venous thromboembolism (VTE) in each age range. ${ }^{*} p<0.05$. Patients aged $30-39$ years showed a higher VTE rate $(5.8 \%)$ in the pandemic period than that in 2019 period $(3.9 \%)$

Table 2 Characteristics of venous thromboembolism in the pandemic period and in the same period of 2019

\begin{tabular}{|c|c|c|c|}
\hline & $\begin{array}{l}\text { Pandemic } \\
(\mathrm{n}=1458)\end{array}$ & $\begin{array}{l}2019 \\
(n=1900)\end{array}$ & $p$ value \\
\hline Clinical manifestations & & & 0.686 \\
\hline Limb symptoms alone, $\mathrm{n}(\%)$ & $1297(89.0)$ & $1672(88.0)$ & \\
\hline $\begin{array}{l}\text { Non-limb symptoms alone, } \\
\mathrm{n}(\%)\end{array}$ & $137(9.4)$ & $195(10.3)$ & \\
\hline Both, n (\%) & $24(1.6)$ & $33(1.7)$ & \\
\hline VTE & & & 0.737 \\
\hline Only DVT, n (\%) & $1142(78.3)$ & $1494(78.6)$ & \\
\hline Only PE, n (\%) & $26(1.8)$ & $40(2.1)$ & \\
\hline DVT and PE, n (\%) & $290(19.9)$ & $366(19.3)$ & \\
\hline \multicolumn{4}{|l|}{ Treatment } \\
\hline Anticoagulation alone, $\mathrm{n}(\%)$ & $488(33.5)$ & $698(36.7)$ & 0.050 \\
\hline IVCF implantation, n (\%) & $970(66.5)$ & $1201(63.2)$ & 0.046 \\
\hline Additional IR therapy, n (\%) & $643(44.1)$ & 797 (41.9) & 0.211 \\
\hline Short-outcome on discharge & & & 0.252 \\
\hline Remission, $\mathrm{n}(\%)$ & $1403(96.2)$ & 1835 (96.6) & \\
\hline Unremission, n (\%) & $47(3.2)$ & $48(2.5)$ & \\
\hline Death, n $(\%)$ & $8(0.5)$ & $17(0.9)$ & \\
\hline
\end{tabular}

$V T E$ venous thromboembolism, $D V T$ deep venous thrombosis, $P E$ pulmonary embolism, $I V C F$ inferior vena cava filter, $I R$ interventional radiology

patients without COVID-19 but with risk factors to increase mobility, and even administer pharmacologic prophylaxis after risk assessment. Telemedicine and e-visits were preferable to minimize the risks of in-person interactions between healthcare worker and patient [2].

Under the panic of COVID-19, it showed that patients were more likely to choose aggressive treatment. The rate of IVCF implantation and additional interventional therapy increased in the pandemic period. And only $33.5 \%$ patients were treated with anticoagulation alone in the pandemic period, which was lower than that in 2019.

The findings in this study have some crucial implications for health-care resource planning and preventive strategies. To our knowledge, it is the largest study to date that examined how the pandemic affects the prevalence of VTE in patients without COVID-19 in China. The study has some limitations. First, because of its retrospective nature and limitation of the data available (derived predominantly from the radiology departments), and those with VTE did not go to the hospitals were not included, a potential exists for misrepresentation of national hospitalizations may exist. Second, lack of information (duration, severity, follow-up, etc.) limited further evaluation. Third, the pandemic period (from January 24th to April 16th) was not divided into different stages reported by other studies [21, 22]. Even so, we tried to send an important message as Dr. Rosenbaum called for, "Covid or no Covid, we are still here to care for you" [23]. Promoting healthy living for future pandemic protection should be considered [24].

\section{Conclusions}

This study provides insights into the disease burden of VTE in non-COVID-19 population in China. The pandemic increased the VTE risk in patients with decreased activity among the non-COVID-19 population. Patients with comorbidities, especially diabetes, have a significant higher risk of VTE during the pandemic. Therefore, we would like to call for attentions to non-COVID-19 people during the pandemic.

Supplementary Information The online version contains supplementary material available at https://doi.org/10.1007/s11239-021-02442-2.

Acknowledgements Thanks to all the doctors from 94 centers in the mainland of China who helped to collect the data: Chen Wang (Gansu Provincial Hospital of TCM); Chuansheng Zheng (Union Hospital, Tongji Medical College, Huazhong University of Science and Technology); Fandong Li (The Second Hospital of Shandong University); Feng Wang (The First Affiliated Hospital of Dalian Medical University); Gang Cao (Lianyungang First People's Hospital); Gang Sun (960th Hospital of PLA); Guodong Chen (Guangzhou First People's Hospital); Guohui Xu (Sichuan Cancer Hospital and Institute, Sichuan Cancer Center); Guowen Yin (Cancer Hospital of Jiangsu Province); Guozuo Xiong (The Second Affiliated Hospital, University of South China); Haibo Shao (The First Hospital of China Medical University); Haiying Jin (Huaihe Hospital Henan University); Hanwei Chen (Guangzhou Panyu Central Hospital); Hao Xu (Affiliated Hospital of Xuzhou Medical University); Hong Shan, Pengfei Pang (The Fifth Affiliated Hospital of Sun Yat-sen University); Hongjun Hou (Weihai Central Hospital); Hongtao Niu (Qinhuangdao First Hospital); Hongwen Zhang (Affiliated Nanhua Hospital, University of South China); Hongxin Zhang (Tangdu Hospital, Air Force Military Medical University); Hongyao Hu (Renmin Hospital of Wuhan University); Hua Xiang (Hunan 
Provincial People's Hospital); Huanjun Chen (Yanan Hospital of Kunming City); Hui Zhao (Affiliated Hospital of Nantong University); Jian $\mathrm{Xu}$ (Nanjing General Hospital of the Nanjing Military Command); Jian You (Guiqian International General Hospital); Jianguo Wang (Shuyang People's Hospital); Jianhua Huang (Xiangya Hospital, Central South University); Jiansong Ji (Lishui Central Hospital); Jianwei Jiang (Wuxi Third People's Hospital); Jiaping Li (The First Affiliated Hospital of Sun Yat-sen University); Jiasheng Huang (The Affiliated Huai' an No.1 People's Hospital); Jing Zhang (Guangzhou Women and Children's Medical Centre); Jinguo Cui (Bethune International Peace Hospital);Jinqi Huang, Huang Chen (The First Hospital of Putian City); Kangshun Zhu (The Second Affiliated Hospital of Guangzhou Medical University); Kunqian Chen (Qujing First People's Hospital); Lefeng Qu (Shanghai Changzheng Hospital); Lei Chen (Suzhou Municipal Hospital); Lei Song (The Second Affiliated Hospital of Dalian Medical University); Lei Yu (the People's Hospital of Guangxi Zhuang Autonomous Region); Ligong Lu (Zhuhai People's Hospital); Linan Du (The Second Affiliated Hospital of The Medical University of Anhui); Long Jin (Beijing Friendship Hospital); Maoquan Li (Tenth People's Hospital of Tongji University); Ming Che (Gansu Provincial Hospital); Ming Huang (Yunnan Tumor Hospital); Mugen Zhang (the 92nd Hospital of Chinese PLA); Penghua Lv (Northern Jiangsu People's Hospital); Ping Xie (Sichuan Provincial People's Hospital); Po Yang (the Fourth Affiliated Hospital of Harbin Medical University); Qing Li (Affiliated Hospital of Xiangnan University); Rongshu Shi, Feng Li (Affiliated Hospital of Zunyi Medical University); Ruijun Ren (Huhhot First Hospital); Shaoxin Yao (Tangshan Workers' Hospital); Shi Zhou (The Affiliated Hospital of Guizhou Medical University); Shiwu Yin (The Second People's Hospital of Hefei); Shuguang Zhang (The First Affiliated Hospital of Shandong First Medical University); Shunchi $\mathrm{Xu}$ (The Fourth Hospital of Changsha City the Fourth Hospital of Changsha); Tongqing Xue (Huai' an Chuzhou Hospital); Wanyin Shi (The First Affiliated Hospital of Anhui Medical University); Wei Li (Jingmen No.2 People's Hospital); Weidong Wang, Wei Ding (Wuxi First People's Hospital); Weifu Lv (Anhui Provincial Hospital); Wendong Cao(Bethune Hospital Shanxi Medical University); Wenge Xing, Haipeng Yu (Tianjin Medical University Cancer Institute and Hospital); Wenhui Wang (Lanzhou University First Affiliated Hospital); Xiangzhong Huang (Jiangyin People's Hospital); Xiao Li (Cancer Hospital Chinese Academy of Medical Sciences); Xiaofei Sun (The Mineral General Hospital of Xuzhou); Xiaofeng He, Jianbo Zhao (Nanfang Hospital Southern Medical University), Xiaoli Zhu (The First Affiliated Hospital of Soochow University); Xiaolin Zhang (Yichang Central Hospital); Xiaoming Chen (Guangdong Provincial People's Hospital); Xindong Fan (Shanghai Ninth People's Hospital); Xinlin Yu (Second People's Hospital of Gansu Province); Xiujun Zhang (Tianjin Nankai Hospital); Xixiang Yu (Wenzhou People's Hospital); Xudong Chen (Shenzhen People's Hospital); Xuejun Zhang/Sheng Xu (Affiliated Hospital of Inner Mongolia Medical University); Xuya Zhao (The Affiliated Cancer Hospital of Guizhou Medical University); Yamin Liu (First Affiliated Hospital of Xi' an Jiaotong University); Yang Weizhu (Affiliated Union Hospital of Fujian Medical University); Yanming Lei (The Tibet Autonomous Region People's Hospital); Yanrong Zhang (Third Hospital of Hebei Medical University); Yilong Ma (Affiliated Tumor Hospital of Guangxi Medical University); Yinghua Zou, Jian Wang (Peking University First Hospital); Yingsheng Cheng, Yueqi Zhu (Shanghai Jiao Tong University Affiliated Sixth People's Hospital); Zhaoyu Liu (Shengjing Hospital of China Medical University); Zhenhai Di (Affiliated Hospital of Jiangsu University); Zhenyu Dai, Wenhui Li (Yancheng Third People's Hospital); Zhongmin Wang (Luwan Branch, Ruijin Hospital, Shanghai Jiaotong University); Zhuting Fang (Fujian Provincial Hospital).

Funding Nanjing Medical University Science and Technology Development Funding (General Projects): NMUB2019144.

\section{Declarations}

Conflict of interest All authors declare that they have no conflict of interest.

\section{References}

1. Organization WH Coronavirus Disease 2019 (COVID-19) situation report-59. https://www.who.int/docs/default-source/coron aviruse/situation-reports/20200319-sitrep-59-covid-19.pdf? sfvrsn=c3dcdef9_2.

2. Bikdeli B, Madhavan MV, Jimenez D et al (2020) COVID-19 and thrombotic or thromboembolic disease: implications for prevention, antithrombotic therapy, and follow-up. J Am Coll Cardiol 75:2950-2973

3. Driggin E, Madhavan MV, Bikdeli B et al (2020) Cardiovascular considerations for patients, health care workers, and health systems during the COVID-19 pandemic. J Am Coll Cardiol 75:2352-2371

4. Tang N, Li D, Wang X, Sun Z (2020) Abnormal coagulation parameters are associated with poor prognosis in patients with novel coronavirus pneumonia. J Thromb Haemost 18:844-847

5. Cui S, Chen S, Li X et al (2020) Prevalence of venous thromboembolism in patients with severe novel coronavirus pneumonia. J Thromb Haemost 18:1421-1424

6. Klok FA, Kruip MJHA, van der Meer NJM et al (2020) Incidence of thrombotic complications in critically ill ICU patients with COVID-19. Thromb Res 191:145-147

7. Melov SJ, Hitos K (2018) Venous thromboembolism risk and postpartum lying-in: acculturation of Indian and Chinese women. Midwifery 58:13-18

8. Salim S, Zarrouk M, Elf J et al (2019) Clinical implications of different risk factor profiles in patients with mesenteric venous thrombosis and systemic venous thromboembolism: a population-based study. J Thromb Thrombolysis 47:572-577

9. Spencer FA, Gore JM, Lessard D et al (2008) Venous thromboembolism in the elderly a community-based perspective. Thromb Haemost 100:780-788

10. Næss IA, Christiansen SC, Romundstad P et al (2007) Incidence and mortality of venous thrombosis: a population-based study. J Thromb Haemost 5:692-699

11. Tritschler T, Aujesky D (2017) Venous thromboembolism in the elderly: a narrative review. Thromb Res 155:140-147

12. Cheah PY, Pan-ngum W, Poomchaichote T et al (2020) Social, ethical and behavioural aspects of COVID-19. Wellcome Open Res 5:1-13

13. Bourdas DI, Zacharakis ED (2020) Evolution of changes in physical activity over lockdown time: physical activity datasets of four independent adult sample groups corresponding to each of the last four of the six COVID-19 lockdown weeks in Greece. Data $\mathrm{Br}$ 32:106301

14. Engbers MJ, Blom JW, Cushman M et al (2014) The contribution of immobility risk factors to the incidence of venous thrombosis in an older population. J Thromb Haemost 12:290-296

15. Pang H, Wang L, Liu J et al (2018) The prevalence and risk factors of venous thromboembolism in hospitalized patients with acute exacerbation of chronic obstructive pulmonary disease. Clin Respir J 12:2573-2580

16. Ye F, Stalvey C, Khuddus MA et al (2017) A systematic review of mobility/immobility in thromboembolism risk assessment models for hospitalized patients. J Thromb Thrombolysis 44:94-103 
17. Sakkinen PA (2000) Clustering of procoagulation, inflammation, and fibrinolysis variables with metabolic factors in insulin resistance syndrome. Am J Epidemiol 152:897-907

18. Bai J, Ding X, Du X et al (2015) Diabetes is associated with increased risk of venous thromboembolism: a systematic review and meta-analysis. Thromb Res 135:90-95

19. Ghram A, Briki W, Mansoor H et al (2020) Home-based exercise can be beneficial for counteracting sedentary behavior and physical inactivity during the COVID-19 pandemic in older adults. Postgrad Med 00:1-12

20. Jiménez-Pavón D, Carbonell-Baeza A, Lavie CJ (2020) Physical exercise as therapy to fight against the mental and physical consequences of COVID-19 quarantine: special focus in older people. Prog Cardiovasc Dis 63:386-388

21. Shen Y, Cui Y, Li N et al (2020) Emergency responses to Covid19 outbreak: experiences and lessons from a general hospital in Nanjing, China. Cardiovasc Intervent Radiol 43:810-819
22. Zhu H-D, Zeng C-H, Lu J, Teng G-J (2020) COVID-19: what should interventional radiologists know and what can they do? J Vasc Interv Radiol 31:876-881

23. Rosenbaum L (2020) The untold toll - the pandemic's effects on patients without Covid-19. N Engl J Med 382:2368-2371

24. Arena R, Lavie CJ (2020) The global path forward-Healthy Living for Pandemic Event Protection (HL - PIVOT). Prog Cardiovasc Dis 64:96-101

Publisher's Note Springer Nature remains neutral with regard to jurisdictional claims in published maps and institutional affiliations. 